\title{
ON THE MIXTURE OF WEIGHTED EXPONENTIAL AND WEIGHTED GAMMA DISTRIBUTION
}

\section{TASWAR IQBAL*, MUHAMMAD ZAFAR IQBAL}

\author{
Department of Mathematics and Statistics, University of Agriculture Faisalabad, Faisalabad, Pakistan
}

*Corresponding author: therisers1@gmail.com

\begin{abstract}
In practice, finite mixture models were often used to fit various type of observed phenomena, specifically those which are random in nature. In this paper, a finite mixture model based on weighted versions of exponential and gamma distribution is considered and studied. Some mathematical properties of the resulting model are discussed including moment generating function, skewness, kurtosis, survival function, hazard rate function, stochastic ordering, order statistics, Bonferroni and Lorenz curves, Renyi entropy measure and estimation of the model parameters. Two real-life data applications from different fields exhibit the fact that in certain situations, the proposed mixture model might be a better alternative than the existing popular models.
\end{abstract}

\section{INTRODUCTION}

The mixture distributions over time have provided a mathematical based way to model a wide range of random phenomena. The mixture models are effective and flexible to analyze and interpret random situations in a possibly heterogeneous populations. In many situations, observed data may be assumed to have come from a mixture population of two or more distributions. The mixture models are used in medicine, psychology, finance, engineering, fisheries research, economics, life testing and reliability among others. In this paper, we perpend a finite mixture of two continuous distributions, a one-parameter exponential distribution and a two-parameter gamma distribution. In mixture model, the distribution of interest is

Received January $15^{\text {th }}, 2020$; accepted February $10^{\text {th }}, 2020$; published May $1^{\text {st }}, 2020$.

2010 Mathematics Subject Classification. 60E05, 62E15.

Key words and phrases. finite mixture models; weighted exponential distribution; weighted gamma distribution.

(C)2020 Authors retain the copyrights of their papers, and all open access articles are distributed under the terms of the Creative Commons Attribution License. 
modeled as a mixture of two or more distributions in varying proportions. Thus a mixture model can be used to model complex situations through proper choice of its mixing components. It can handle situations where a single parametric family is unable to provide a satisfactory model for local variation in the observed data. The flexibility and high degree of accuracy of finite mixture models have been the main reason for their successful applications in a wide range of fields. The concept of finite mixture distribution was pioneered by Newcomb [1] as a model for outliers. However, the credit for the introduction of statistical modeling using finite mixture of distributions goes to Pearson [2] while applying the technique in an analysis of crab morphometry data provided by Weldon [3] and [4] For a comprehensive survey, readers are referred to Titterington et al. [5] Lindsay [6] Bohning [18] and McLachlan and Peel [19] and the references therein. The main objective here is to induce greater flexibility in modeling various types of data, specially in situations where these individual distributions fail to adequately fit the data separately. In the recent years a number of new life time distributions have been developed and proposed by many researchers which are in general modification or an extension or generalization of Lindley distribution. The range of such distributions include Modified One Parameter Lindley distribution [7], Improved Second-Degree Lindley distribution [8], Amarendra distribution [9], Sujatha distribution [10], Shankar distribution [11] and Akash distribution [12]. Each model has its own limitations and advantages for modeling the lifetime data set. As an extension of these models, in the context to find a new distribution which is more flexible we have introduced a new continuous one-parameter distribution. The new proposed model is a two-component mixture of length biased exponential distribution with parameter $\theta$ and length biased gamma distribution with shape parameter 2 and scale parameter $\theta$ with their mixing proportions $\frac{\theta}{\theta+1}$ and $\frac{1}{\theta+1}$ respectively.

Suppose we have the following model

$$
f(x)=p f_{1}(x)+(1-p) f_{2}(x)
$$

where $f_{j}(x), j=1,2$ are densities. Clearly, $p=\frac{\theta}{\theta+1}$ and $1-p=\frac{1}{\theta+1}$, are mixture weights $(0<p<1)$, and $f(x)$ indeed a valid density. Next, we have

$$
\begin{gathered}
f_{1}(x)=\theta^{2} x e^{-\theta x} \\
f_{2}(x)=\frac{\theta^{3}}{2} x^{2} e^{-\theta x}
\end{gathered}
$$

Our weighted distribution will have density

$$
f(x)=\frac{\theta^{3}}{\theta+1}\left[1+\frac{x}{2}\right] x e^{-\theta x} \quad x>0, \theta>0
$$

The density in (1.1) is called the mixture of weighted exponential and weighted gamma (hereafter MWEG in short) distribution. The cumulative distribution function of MWEG distribution can be obtained as

$$
F(x)=1-\frac{\left[\theta^{2} x^{2}+(\theta+1) 2 \theta x+2(\theta+1)\right] e^{-\theta x}}{2(\theta+1)} \quad x>0, \theta>0
$$




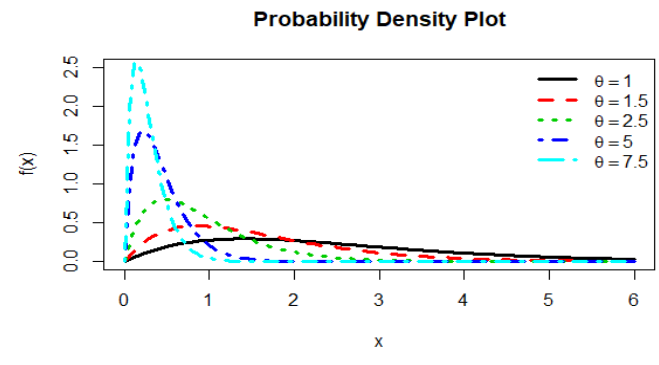

(a)

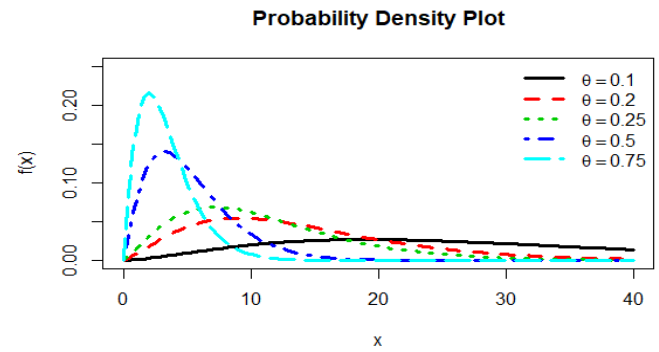

(b)

Figure 1. Probability density function of MWEG distribution at different values of $\theta$



(a)

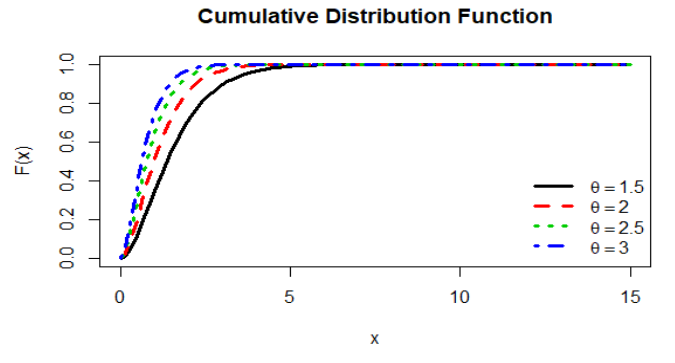

(b)

Figure 2. Cumulative distribution function of MWEG distribution at different values of $\theta$

\section{Properties of the MWEG Distribution}

The mathematical properties of the proposed MWEG distribution are discussed as follows

2.1. Hazard Function. The hazard function is defined as the ratio of probability density function to the survival function, it is also known as the hazard rate or force of mortality. The hazard function associated with the MWEG distribution is

$$
\begin{gathered}
h_{f}(x)=\frac{f(x)}{1-F(x)} \\
h_{f}(x)=\frac{2 \theta^{3} x\left(1+\frac{x}{2}\right)}{\left(\theta^{2} x^{2}+\left(2 \theta^{2}+2 \theta\right) x+2 \theta+2\right)} \\
h_{f}(x)=\frac{\theta^{3} x(2+x)}{\theta^{2} x^{2}+(\theta+1) 2 \theta x+2(\theta+1)}
\end{gathered}
$$




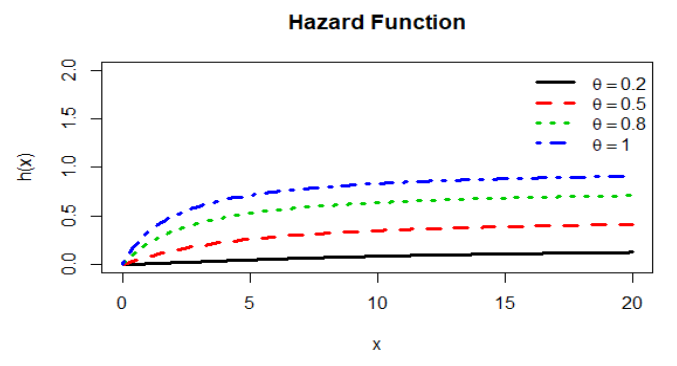

(a)

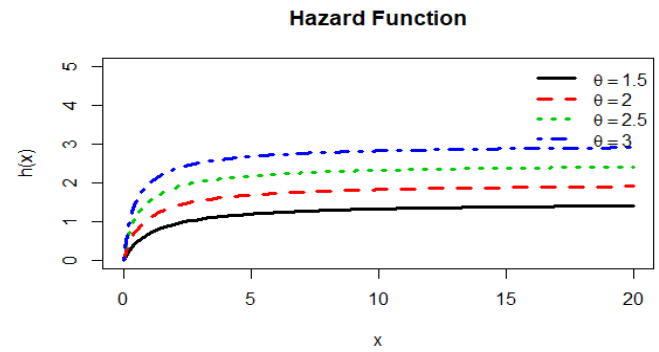

(b)

FiguRE 3. Hazard function of MWEG distribution at different values of $\theta$

2.2. Survival Function. Let $X$ be the non-negative random variable with pdf, $f(x)$ given by (1.1). The reliability function $\mathrm{S}(\mathrm{x})$ corresponding to the finite mixture of 2 components of (1.1) is given by

$$
\begin{gathered}
S(x)=p S_{1}(x)+(1-p) S_{2}(x) \\
S(x)=\frac{\left[\theta^{2} x^{2}+(\theta+1) 2 \theta x+2(\theta+1)\right] e^{-\theta x}}{2(\theta+1)}
\end{gathered}
$$

Where $S_{j}(x)$, is the reliability function corresponding to the $\mathrm{j}$-th component in the mixture, $\mathrm{j}=1,2$. One can write the hazard rate function of a mixture in terms of the hazard rate functions of the two components as follows.

$$
h_{f}(x)=B(x) h_{1}(x)+[1-B(x)] h_{2}(x), \text { Where } B(x)=p S_{1}(x) /\left[p S_{1}(x)+(1-p) S_{2}(x)\right]
$$

and $h_{j}(x)$ are hazard rate function for the $\mathrm{j}$-th component, $\mathrm{j}=1,2$. On differentiating the hazard function, we get

$$
h_{f}^{\prime}(x)=B(x) h_{1}^{\prime}(x)+(1-B(x)) h_{2}^{\prime}(x)-B(x)(1-B(x))\left(h_{1}(x)-h_{2}(x)\right)^{2}
$$

Where prime denote the derivatives with respect to x. Now, from the above, it follows that if $h_{j}^{\prime}(x)<0$, for all $x,(j=1,2)$ then $h^{\prime}(x)<0$, for all $\mathrm{x}$. Therefore, a mixture with decreasing hazard rate components has decreasing hazard rate. However, if the components have increasing hazard rates, their mixture need not have increasing hazard rate.

2.3. Moments and Related Measures. Moments plays a very important role to understand some important features of a distribution. In statistics, moments can be used to study central tendency, dispersion, skewness and kurtosis of any distribution.

The moment generating function of MWEG distribution is

$$
\begin{aligned}
& M_{x}(t)=\frac{\theta^{3}}{\theta+1} \int_{0}^{\infty}\left[1+\frac{x}{2}\right] x e^{-(\theta-t) x} d x \\
& M_{x}(t)=\frac{\theta^{3}(\theta+t+1)}{(\theta+1)\left(\theta^{3}+3 t \theta^{2}+3 t^{2} \theta+t^{3}\right)}
\end{aligned}
$$




$$
M_{x}(t)=\frac{\theta^{3}(\theta+t+1)}{(\theta+1)(\theta+t)^{3}}
$$

By using the equation (2.2) the first four moments about the origin of MWEG distribution are given by

$$
\begin{aligned}
& \mu_{1}^{\prime}=\frac{2 \theta+3}{\theta(\theta+1)} \\
& \mu_{2}^{\prime}=\frac{6(\theta+2)}{\theta^{2}(\theta+1)} \\
& \mu_{3}^{\prime}=\frac{12(2 \theta+5)}{\theta^{3}(\theta+1)} \\
& \mu_{4}^{\prime}=\frac{120(\theta+3)}{\theta^{4}(\theta+1)}
\end{aligned}
$$

By using the relationship between moments about origin and moments about mean, the moments about mean of MWEG distribution are given by

$$
\begin{gathered}
\mu_{2}=\frac{2 \theta^{2}+6 \theta+3}{\theta^{2}(\theta+1)^{2}} \\
\mu_{3}=\frac{2\left(2 \theta^{3}+9 \theta^{2}+9 \theta+3\right)}{\theta^{3}\left(\theta^{3}+3 \theta^{2}+3 \theta+1\right)} \\
\mu_{4}=\frac{\left.24 \theta^{4}+144 \theta^{3}+252 \theta^{2}+180 \theta+45\right)}{\theta^{4}\left(\theta^{4}+4 \theta^{3}+6 \theta^{2}+4 \theta+1\right)}
\end{gathered}
$$

Skewness and Kurtosis plays a vital role in explaining the shape and tail property of a distribution. The expressions of Skewness and Kurtosis are given by

$$
\begin{gathered}
\sqrt{\beta_{1}}=\frac{\mu_{3}}{\mu_{2}^{3 / 2}}=\frac{2\left(2 \theta^{3}+9 \theta^{2}+9 \theta+3\right)}{\left(2 \theta^{2}+6 \theta+3\right)^{3 / 2}} \\
\beta_{2}=\frac{\mu_{4}}{\mu_{2}^{2}}=\frac{3\left(8 \theta^{4}+48 \theta^{3}+84 \theta^{2}+60 \theta+15\right)}{\theta^{2}(\theta+1)^{2}\left(2 \theta^{2}+6 \theta+3\right)}
\end{gathered}
$$

The index of dispersion and coefficient of variation are thus obtained as

$$
\begin{gathered}
C . V=\frac{\sigma}{\mu_{1}^{\prime}}=\frac{\sqrt{2 \theta^{2}+6 \theta+3}}{(2 \theta+3)} \\
\gamma=\frac{\sigma^{2}}{\mu_{1}^{\prime}}=\frac{2 \theta^{2}+6 \theta+3}{\theta(\theta+1)(2 \theta+3)}
\end{gathered}
$$

The condition under which MWEG distribution is over-dispersed, under-dispersed, and equi-dispersed has been given along Amarendra, Ishita, Akash, Lindley and exponential distributions in table 1 
TABLE 1. Over-dispersion, under-dispersion and equi-dispersion MWEG, Ishita, Amarendra, Akash, Lindley and exponential distributions for parameter $\theta$.

\begin{tabular}{c|c|c|c}
\hline Distribution & Over-dispersion & Under-dispersion & Equi-dispersion \\
\hline MWEG & $\theta<1.10073$ & $\theta>1.10073$ & $\theta=1.10073$ \\
Ishita & $\theta<1.535653152$ & $\theta>1.535653152$ & $\theta=1.535653152$ \\
Amarendra & $\theta<1.525763580$ & $\theta>1.525763580$ & $\theta=1.525763580$ \\
Akash & $\theta<1.515400063$ & $\theta>1.515400063$ & $\theta=1.515400063$ \\
Lindley & $\theta<1.170086487$ & $\theta>1.170086487$ & $\theta=1.170086487$ \\
Exponential & $\theta<1$ & $\theta>1$ & $\theta=1$ \\
\hline
\end{tabular}

2.4. Mean Residual Life Function. The mean residual function of the MWEG distribution is given as

$$
\begin{gathered}
m(x)=\frac{1}{1-F(x)} \int_{x}^{\infty}[1-F(t)] d t \\
m(x)=\frac{2(\theta+1)}{\left[\theta^{2} x^{2}+(\theta+1) 2 \theta x+2(\theta+1)\right] e^{-\theta x}} \int_{x}^{\infty}\left[\frac{\theta^{2} t^{2}+(\theta+1) 2 \theta t+2(\theta+1)}{2(\theta+1)}\right] e^{-\theta t} d t \\
m(x)=\frac{\theta^{2} x^{2}+(\theta+2) 2 \theta x+2(2 \theta+3)}{\theta\left[\theta^{2} x^{2}+(\theta+1) 2 \theta x+2(\theta+1)\right]}
\end{gathered}
$$

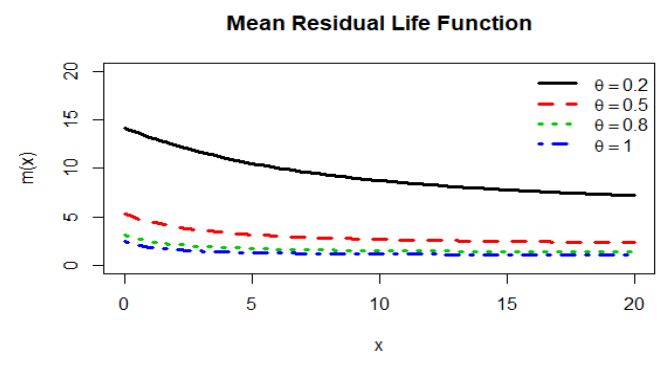

(a)

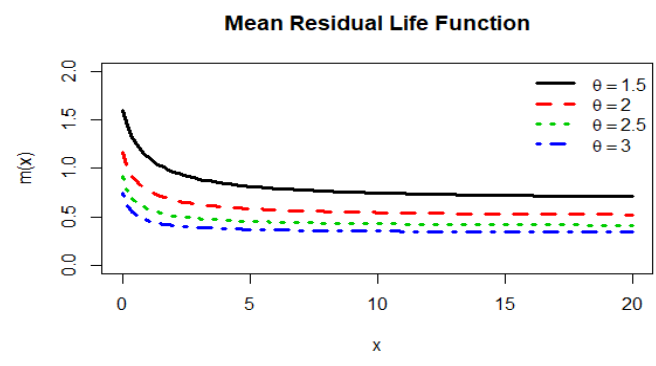

(b)

Figure 4. Mean residual life function of MWEG distribution at different values of $\theta$

2.5. Stochastic Orderings. In probability theory, stochastic ordering is considered an important tool for assessing the comparative behavior of a positive continuous random variable. A random variable $X$ is said to be smaller than a random variable $Z$, in the

i. Stochastic order $\left(X \leq_{s t} Z\right)$, if $F_{X}(x) \geq F_{Z}(x)$ for all $x$.

ii. Hazard rate order $\left(X \leq_{h r} Z\right)$, if $h_{X}(x) \geq h_{Z}(x)$ for all $x$.

iii. Mean residual life order $\left(X \leq_{m r l} Z\right)$, if $m_{X}(x) \leq m_{z}(x)$ for all $x$. 
iv. Likelihood ratio order $\left(X \leq_{l r} Z\right)$, if $\frac{f_{X}(x)}{f_{Z}(x)}$ decreases in $x$.

Shaked and Shanthikumar [13] proposed the following well-known results for demonstrating the stochastic ordering of distributions

$$
X \leq_{l r} Z \Rightarrow X \leq_{h r} Z \Rightarrow X \leq_{m r l} Z \Rightarrow X \leq_{s t} Z
$$

In the following theorem, it has shown that MWEG distribution is being ordered with respect to the strongest "Likelihood ratio" ordering

Theorem

Let $X \sim$ MWEG distribution $\left(\theta_{1}\right)$ and $Z \sim$ MWEG distribution $\left(\theta_{2}\right)$, if $\theta_{1} \geq \theta_{2}$, then $X \leq{ }_{l r} Z$ and hence $X \leq_{h r} Z \Rightarrow X \leq_{m r l} Z$ and $X \leq_{s t} Z$

Proof

We have

$$
\begin{gathered}
f_{X}(x)=\frac{\theta_{1}^{3}}{\theta_{1}+1}\left[x+\frac{x^{2}}{2}\right] e^{-\theta_{1} x} \\
f_{z}(x)=\frac{\theta_{2}^{3}}{\theta_{2}+1}\left[x+\frac{x^{2}}{2}\right] e^{-\theta_{2} x} \\
\frac{f_{X}(x)}{f_{z}(x)}=\frac{\frac{\theta_{1}^{3}}{\theta_{2}^{3}}\left[x+\frac{x^{2}}{2}\right] e^{-\theta_{1} x}}{\theta_{2}^{3}+1}\left[x+\frac{x^{2}}{2}\right] e^{-\theta_{2} x} \\
\frac{f_{X}(x)}{f_{z}(x)}=\frac{\theta_{1}^{3}\left(\theta_{2}+1\right)}{\theta_{2}^{3}\left(\theta_{1}+1\right)} e^{-\left(\theta_{1}-\theta_{2}\right) x} \\
\log \left[\frac{f_{x}(x)}{f_{z}(x)}\right]=\log \left[\frac{\theta_{1}^{3}\left(\theta_{2}+1\right)}{\theta_{2}^{3}\left(\theta_{1}+1\right)}\right]-\left(\theta_{1}-\theta_{2}\right) x
\end{gathered}
$$

This implies that

$$
\frac{d}{d x} \log \frac{f_{X}(x)}{f_{z}(x)}=-\left(\theta_{1}-\theta_{2}\right)
$$

Hence for $\theta_{1} \geq \theta_{2}, \frac{d}{d x} \log \frac{f_{x}(x)}{f_{z}(x)}<0$, it means that $X \leq_{l r} Z$ and hence $X \leq_{h r} Z \Rightarrow X \leq_{m r l} Z$ and $X \leq{ }_{s t} Z$

2.6. Order Statistics. Let $X_{1}, X_{2}, X_{3} \ldots X_{n}$ be a random sample of size $n$ from MWEG distribution. Let $X_{(1)}, X_{(2)}, X_{(3)} \ldots X_{(n)}$ denotes the corresponding order statistics. The probability density function and the 
cumulative distribution function of the $K^{t h}$ order statistic, say $Z=X_{(k)}$ are given below

$$
\begin{aligned}
& f_{z}(x)=n\left(\begin{array}{c}
n-1 \\
k-1
\end{array}\right) F(x)^{k-1}(1-F(x))^{n-k} f(x) \\
& \text { and } \\
& F_{z}(x)=\sum_{j=k}^{n} \sum_{l=0}^{n-j}\left(\begin{array}{c}
n \\
j
\end{array}\right)\left(\begin{array}{c}
n-j \\
l
\end{array}\right)(-1)^{l} F(x)^{j+l}
\end{aligned}
$$

Respectively for different values of $k=1, \ldots, n$ Hence the probability density function and cumulative distribution function of $K^{\text {th }}$ order statistics are given as

$$
\begin{aligned}
f_{z}(x)= & \frac{\theta^{3}}{\theta+1}\left[1+\frac{x}{2}\right] x e^{-\theta x} n\left(\begin{array}{c}
n-1 \\
k-1
\end{array}\right) \\
& \left(1-\frac{\left(\theta^{2} x^{2}+\left(2 \theta^{2}+2 \theta\right) x+2 \theta+2\right) e^{-\theta x}}{2(\theta+1)}\right)^{k-1}\left(\frac{\left(\theta^{2} x^{2}+\left(2 \theta^{2}+2 \theta\right) x+2 \theta+2\right) e^{-\theta x}}{2(\theta+1)}\right)^{n-k}
\end{aligned}
$$

It can be written as

$$
f_{z}(x)=\frac{n ! \theta^{3}(2+\theta) x e^{-\theta x}}{2(\theta+1)(n-k) !(k-1) !} \sum_{l=0}^{n-k}\left(\begin{array}{c}
n-k \\
l
\end{array}\right)(-1)^{l}\left(1-\frac{\left.\left(\theta^{2} x^{2}+\left(2 \theta^{2}+2 \theta\right) x+2 \theta+2\right) e^{-\theta x}\right) e^{-\theta x}}{2(\theta+1)}\right)^{k+l-1}
$$

Similarly

$$
F_{z}(x)=\sum_{j=k}^{n} \sum_{l=0}^{n-j}\left(\begin{array}{c}
n-j \\
j
\end{array}\right)\left(\begin{array}{c}
n-j \\
l
\end{array}\right)(-1)^{l}\left(1-\frac{\left(\theta^{2} x^{2}+\left(2 \theta^{2}+2 \theta\right) x+2 \theta+2\right) e^{-\theta x}}{2(\theta+1)}\right)^{j+l}
$$

2.7. Bonferroni and Lorenz Curves. The Bonferroni and Lorenz curves [14] and Bonferroni and Gini indices have applications in many fields like insurance, medicines, demography and also in economics for studying the patterns of income and poverty. The Bonferroni and Lorenz curves may be defined as

$$
\begin{gathered}
B p=\frac{1}{P \mu} \int_{0}^{q} x f(x) d x \\
B p=\frac{1}{P \mu} \int_{0}^{\infty} x f(x) d x-\int_{q}^{\infty} x f(x) d x \\
B(P)=\frac{1}{P \mu}\left[\mu-\int_{q}^{\infty} x f(x) d x\right] \\
B(P)=\frac{1}{P}\left[1-\frac{1}{\mu} \int_{q}^{\infty} x f(x) d x\right]
\end{gathered}
$$

and

$$
L P=\frac{1}{\mu} \int_{0}^{q} x f(x) d x=\frac{1}{\mu}\left[x f(x) d x-\int_{q}^{\infty} x f(x) d x\right]
$$




$$
\begin{gathered}
L(P)=\frac{1}{\mu}\left[\mu-\int_{q}^{\infty} x f(x) d x\right] \\
L(P)=1-\frac{1}{\mu} \int_{q}^{\infty} x f(x) d x
\end{gathered}
$$

Where $E(X)=\mu$ and $q=F^{-1}(P)$ By using (1.1) and (1.2), we can define the Bonfernoni and Gini indices as

$$
\begin{aligned}
& B=1-\int_{0}^{1} B(P) d P \\
& B=1-\int_{0}^{1} B(P) d P
\end{aligned}
$$

and

$$
G=1-2 \int_{0}^{1} L(P) d P
$$

Now by using pdf of MWEG distribution, we get

$$
\begin{gathered}
\int_{q}^{\infty} x f(x) d x=\int_{q}^{\infty} \frac{\theta^{3}}{\theta+1}\left[1+\frac{x}{2}\right] x e^{-\theta x} d x \\
\int_{q}^{\infty} x f(x) d x=\frac{\theta^{3}\left(q^{3}+2 q^{2}\right)+\theta^{2}\left(3 q^{2}+4 q\right)+\theta(6 q+4)+6}{2 \theta(\theta+1)} e^{-\theta q}
\end{gathered}
$$

Using above (2.9) in (2.5) and (2.6), we get

$$
\begin{aligned}
& \frac{1}{\mu} \int_{q}^{\infty} x f(x) d x=\frac{\theta^{3}\left(q^{3}+2 q^{2}\right)+\theta^{2}\left(3 q^{2}+4 q\right)+\theta(6 q+4)+6}{2(2 \theta+3)} e^{-\theta q} \\
& B(P)=\frac{1}{P}\left[1-\frac{\theta^{3}\left(q^{3}+2 q^{2}\right)+\theta^{2}\left(3 q^{2}+4 q\right)+\theta(6 q+4)+6}{2(2 \theta+3)} e^{-\theta q}\right]
\end{aligned}
$$

and

$$
L(P)=1-\frac{\theta^{3}\left(q^{3}+2 q^{2}\right)+\theta^{2}\left(3 q^{2}+4 q\right)+\theta(6 q+4)+6}{2(2 \theta+3)} e^{-\theta q}
$$

By using (2.10) and (2.11) in (2.7) and (2.8), we get

$$
B=1-\frac{\theta^{3}\left(q^{3}+2 q^{2}\right)+\theta^{2}\left(3 q^{2}+4 q\right)+\theta(6 q+4)+6}{2(2 \theta+3)} e^{-\theta q}
$$

and

$$
G=-1+\frac{\theta^{3}\left(q^{3}+2 q^{2}\right)+\theta^{2}\left(3 q^{2}+4 q\right)+\theta(6 q+4)+6}{2 \theta+3} e^{-\theta q}
$$


2.8. Renyi Entropy. Entropy of a random variable $X$ can be defined as a measure of variation of uncertainty. Renyi entropy [15] is considered as a very popular entropy measure. If $X$ is a continuous random variable having probability density function $f(x)$, then Renyi entropy is defined as

$$
T_{R}(y)=\frac{1}{1-\gamma} \log \left\{\int f^{\gamma}(x) d x\right\}
$$

where $\gamma>0$ also $\gamma \neq 1$

Thus, the Renyi entropy for the MWEG distribution ( 1) can be obtained as

$$
\begin{aligned}
& T_{R}(\gamma)=\frac{1}{1-\gamma} \log \int_{0}^{\infty} \frac{\theta^{3 \gamma}}{(\theta+1)^{\gamma}}\left[1+\frac{x}{2}\right]^{\gamma} x^{\gamma} e^{-s \gamma x} d x \\
= & \frac{1}{1-\gamma} \log \left[\frac{\theta^{3 \gamma}}{(\theta+1)^{\gamma}} \int_{0}^{\infty} \sum_{j=0}^{\infty}\left(\begin{array}{c}
\gamma \\
j
\end{array}\right)\left(\frac{x}{2}\right)^{j} x^{\gamma} e^{-\theta \gamma x} d x\right] \\
= & \frac{1}{1-\gamma} \log \left[\frac{\theta^{3 \gamma}}{(\theta+1)^{\gamma}} \sum_{j=0}^{\infty}\left(\begin{array}{c}
\gamma \\
j
\end{array}\right)\left(\frac{x}{2}\right)^{j} \int_{0}^{\infty} x^{\gamma} e^{-\theta \gamma x} d x\right] \\
= & \frac{1}{1-\gamma} \log \left[\frac{\theta^{3 \gamma}}{(\theta+1)^{\gamma}} \sum_{j=0}^{\infty}\left(\begin{array}{c}
\gamma \\
j
\end{array}\right)\left(\frac{1}{2}\right)^{j} \frac{\Gamma(\gamma+j+1)}{(\theta \gamma)^{\gamma+j+1}}\right] \\
= & \frac{1}{1-\gamma} \log \left[\frac{\theta^{2 \gamma-j-1}}{(\theta+1)^{\gamma}} \sum_{j=0}^{\infty} \frac{\Gamma(\gamma+j+1)}{2^{j} \gamma^{\gamma+j+1}}\left(\begin{array}{c}
\gamma \\
j
\end{array}\right)\right]
\end{aligned}
$$

\section{Estimation of Parameters}

This section consists of estimation of the unknown parameters of proposed model by using the methods of moments and maximum likelihood.

3.1. Maximum Likelihood Estimation. Let $X_{1}, X_{2}, X_{3} \ldots X_{n}$ be an iid random sample from MWEG, then the likelihood function of the MWEG distribution is given by,

$$
L=\left(\frac{\theta^{3}}{\theta+1}\right)^{n} \prod_{i=1}^{n}\left[x_{i}+\frac{x^{2} i}{2}\right] e^{-\theta \sum_{i=1}^{n} x_{i}}
$$

and the log-likelihood function can be written as

$$
\begin{gathered}
\ln L=n \ln \left(\frac{\theta^{3}}{\theta+1}\right)+\sum_{i=1}^{n}\left[x_{i}+\frac{x^{2} i}{2}\right]-n \theta \bar{x} \\
\frac{d}{d \theta}(\ln L)=\frac{3 n}{\theta}-\frac{n}{\theta+1}-n \bar{x}
\end{gathered}
$$

Where $\bar{x}$ is the sample mean. We can find the maximum likelihood estimate of $\theta$ by simply equating $\frac{d}{d \theta}(\ln L)=0$, and it can be find by solving the following nonlinear equation

$$
n \bar{x} \theta(\theta+1)-2 n \theta-3 n=0
$$


3.2. Method of Moment. Let $X_{1}, X_{2}, X_{3} \ldots X_{n}$ be a random sample of size $n$ from MWEG distribution. By equating the first population moment about origin to the sample mean, the method of moment estimate $\hat{\theta}$ of $\theta$ is the same as the ML estimate as given in (3.1)

\section{Real Data Application}

The goodness of fit of MWEG distribution has been checked by using several lifetime data sets from engineering and medical science. In this section, we have used two real-life data sets to compare the goodness of fit by using ML estimate of the parameter of MWEG distribution with the exponential, Akash, Lindley, Ishita and Modified One Parameter Lindley distributions and have proved that MWEG distribution provides better estimate for modeling lifetime data sets as compared to its competing models.

Data Set 1: The following dataset acts the breaking stress of carbon fibers of $50 \mathrm{~mm}$ in length Nichols and Padgett [16]

$3.70,2.12,2.95,4.70,1.25,3.22,1.69,3.27,2.87,1.47,3.11,3.65,2.74,3.15,2.97,2.03,4.38,3.39,3.28,3.09$, $1.87,3.15,4.90,4.42,2.73,1.08,3.39,1.89,1.84,2.81,4.20,3.33,2.55,3.31,1.57,2.41,2.50,2.56,2.96,2.88$, $0.39,3.68,2.48,0.85,1.61,3.31,2.67,3.19,3.60,1.80,2.35,2.82,2.05,3.65,3.75,2.43,2.79,2.85,2.93,3.22$, $3.11,2.53,2.55,2.59,2.03,1.61$

Data set 2: The following data represent the survival times (in days) of 72 guinea pigs infected with virulent tubercle bacilli, observed and reported by Bjerkedal [17]. The data are as follows:

$0.1,0.33,0.44,0.56,0.59,0.72,0.74,0.77,0.92,0.93,0.96,1,1,1.02,1.05,1.07,1.07,1.08,1.08,1.08,1.09$, $1.12,1.13,1.15,1.16,1.2,1.21,1.22,1.22,1.24,1.3,1.34,1.36,1.39,1.44,1.46,1.53,1.59,1.6,1.63,1.63,1.68$, $1.71,1.72,1.76,1.83,1.95,1.96,1.97,2.02,2.13,2.15,2.16,2.22,2.3,2.31,2.4,2.45,2.51,2.53,2.54,2.54$, $2.78,2.93,3.27,3.42,3.47,3.61,4.02,4.32,4.58,5.55$

In order to compare MWEG, exponential, Lindley, Akash, Ishita and modified one parameter Lindley distributions, values of $-2 \ln L, A I C$ (Akaike Information Criterion), AICC (Akaike Information Criterion Corrected), BIC (Bayesian Information Criterion) and K-S Statistic ( Kolmogorov-Smirnov Statistic) for two real data sets have been computed and presented in the following table. 
TABle 2. $M L E$ 's,-2lnL, AIC, AICC, BIC, and $K$-S Statistics of the fitted distributions of

\begin{tabular}{|c|c|c|c|c|c|c|c|c|}
\hline & Model & $M L E$ of $\hat{\theta}$ & $S E(\hat{\theta})$ & $-2 \ln L$ & $A I C$ & $C A I C$ & $B I C$ & $K-S$ \\
\hline \multirow{6}{*}{  } & MWEG & 1.2832 & 0.1751 & 50.8754 & 52.8754 & 53.0976 & 53.8711 & 0.3058 \\
\hline & MOPLD & 1.5213 & 0.1523 & 59.7066 & 61.7066 & 61.9288 & 62.7023 & 0.3569 \\
\hline & Ishita & 1.0948 & 0.1217 & 60.1647 & 62.1647 & 62.3869 & 63.1604 & 0.3507 \\
\hline & Exponential & 0.5263 & 0.1177 & 65.4742 & 67.6742 & 67.8964 & 68.6699 & 0.4395 \\
\hline & Lindely & 0.8162 & 0.1361 & 60.4991 & 62.4991 & 62.7213 & 63.4948 & 0.3911 \\
\hline & Akash & 1.1569 & 0.1456 & 59.5226 & 61.5226 & 61.7448 & 62.5183 & 0.3705 \\
\hline \multirow{6}{*}{ 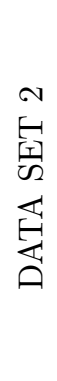 } & MWEG & 1.3697 & 0.0989 & 193.103 & 195.102 & 195.16 & 197.379 & 0.153 \\
\hline & MOPLD & 1.5852 & 0.0839 & 220.004 & 222.004 & 222.061 & 224.281 & 0.231 \\
\hline & Ishita & 1.1598 & 0.0677 & 216.632 & 218.632 & 218.689 & 220.909 & 0.229 \\
\hline & Exponential & 0.5655 & 0.0666 & 226.074 & 228.074 & 228.131 & 230.351 & 0.295 \\
\hline & Lindely & 0.8683 & 0.0766 & 213.857 & 215.857 & 215.914 & 218.134 & 0.247 \\
\hline & Akash & 1.2159 & 0.081 & 214.678 & 216.678 & 216.735 & 218.954 & 0.234 \\
\hline
\end{tabular}

The best distribution corresponds to lower values of $-2 \ln L, A I C, A I C C, B I C$ and $K-S$ statistic. It can be easily seen from above table that MWEG distribution provide better fit as compare to exponential, Lindley, Akash, Ishita and modified one parameter Lindley distributions.

\section{Conclusion}

In this paper, we consider a simple mixture of two absolutely continuous distributions weighted exponential and weighted gamma distribution. Some structural properties of the resulting distribution are discussed. The resulting model appears to be a reasonable choice in the sense of modeling lifetime data sets, in particular, where the popular choices (e.g., Exponential, gamma, Lindley, Ishita and/or Akash distribution) fail to adequately model the observed phenomena. We sincerely hope that this mixture model will find many more applications in different fields affecting human life.

Conflicts of Interest: The author(s) declare that there are no conflicts of interest regarding the publication of this paper.

\section{REFERENCES}

[1] S. Newcomb, A generalized theory of the combination of observations so as to obtain the best result. Amer. J. Math. 8(4)(1886), 343-366.

[2] K. Pearson, Contributions to the mathematical theory of evolution. Philos. Trans. R. Soc. Lond., A. 185 (1894), 71-110. 
[3] W. F. R. Weldon, I. Certain correlated variations in crangon vulgaris. Proceedings of the Royal Society of London, 51(308$314)(1892), 1-21$.

[4] W. F. R. Weldon, II. On certain correlated variations in Carcinus mænas. Proceedings of the Royal Society of London, 54(326-330)(1894), 318-329.

[5] D. M. Titterington, A. F. Smith, and U. E. Makov, Statistical analysis of finite mixture distributions. Wiley, (1985).

[6] B. G. Lindsay, Mixture models: theory, geometry and applications. In NSF-CBMS regional conference series in probability and statistics (pp. i-163). Institute of Mathematical Statistics and the American Statistical Association, (1995).

[7] S. Karuppusamy, V. Balakrishnan, and K. Sadasivan, Modified one-Parameter Lindley Distribution and Its Applications. Int. J. Eng. Res. Appl. 8(1)(2018), 50-56.

[8] S. Karuppusamy, V. Balakrishnan, K. Sadasivan, Improved Second-Degree Lindley Distribution and Its Applications. IOSR J. Math. 13(6)(2018), 1-10.

[9] R. Shanker, Amarendra distribution and Its Applications. Amer. J. Math. Stat. 6(1)(2016), 44-56.

[10] R. Shanker, Sujatha distribution and its Applications. Stat. Trans. New Ser. 17(3)(2016), 391-410.

[11] R. Shanker, Shanker distribution and its applications. Int. J. Stat. Appl. 5(6)(2015), 338-348.

[12] R. Shanker, Akash distribution and its applications. Int. J. Probab. Stat. 4(3)(2015), 65-75.

[13] J. G. Shanthikumar, Stochastic orders and their applications. Academic Press, (1994).

[14] C. E. Bonferroni, Elementi di statistica generale,(ristampa con aggiunte): anno accademico 1932/33; Bari, R. Istit. super. di scienze economiche. Gili. (1933).

[15] A. Rényi, On measures of entropy and information. In Proceedings of the Fourth Berkeley Symposium on Mathematical Statistics and Probability, Volume 1: Contributions to the Theory of Statistics. The Regents of the University of California. (1961).

[16] M. D. Nichols and W. J. Padgett, A bootstrap control chart for Weibull percentiles. Qual. Reliab. Eng. Int. 22(2)(2006), $141-151$.

[17] T. Bjerkedal, Acquisition of Resistance in Guinea Pies infected with Different Doses of Virulent Tubercle Bacilli. Amer. J. Hygiene, 72(1)(1960), 130-48.

[18] D. Böhning, Computer-assisted analysis of mixtures and applications: meta-analysis, disease mapping and others (Vol. 81). CRC press, (1999).

[19] G. J. McLachlan, D. Peel, Finite mixture models. John Wiley \& Sons. (2004). 